\title{
Utilization drop-out of intermittent preventive treatment with sulfadoxine-pyrimethamine among pregnant women in North Western Ghana
}

\author{
Anthony Chiaraah ${ }^{1,}$, Ebenezer Owusu-Sekyere ${ }^{2}$ \\ ${ }^{1}$ Department of Economics and Entrepreneurship Development, University for Development Studies, Wa, Ghana \\ ${ }^{2}$ Department of Development Studies, University for Development Studies, Wa, Ghana
}

Email address:

tonyacgh@yahoo.com (A. Chiaraah), oseturbo@yahoo.com.com (E. Owusu-Sekyere)

\section{To cite this article:}

Anthony Chiaraah, Ebenezer Owusu-Sekyere. Utilization Drop-Out of Intermittent Preventive Treatment with Sulfadoxine-Pyrimethamine among Pregnant Women in North Western Ghana. Science Journal of Public Health. Vol. 2, No. 3, 2014, pp. 156-164.

doi: $10.11648 /$ j.sjph.20140203.13

\begin{abstract}
Although studies have shown the efficacy of Intermittent Preventive Treatment (IPT) with Sulfadoxine-pyrimethamine (SP) as a chemo 'prophylaxis' for malaria during pregnancy, the dose completion rate is still low in many communities in Ghana. Since the implementation of the IPT with SP policy on pilot basis in some districts and the scaling-up of the policy in other districts of the Upper West Region (UWR), available statistics reveal a high drop-out rate in the second dose. This study assessed various factors that contribute to the low utilization and completion rate of SP, and elicit suggestion for the improvement of the implementation of the policy. This anthropological study was conducted in the Wa municipality of Ghana. A total of six FGDs were held, 4 with pregnant women and 2 with post natal mothers using a semi structured questionnaire. In addition $17 \mathrm{key}$ informant interviews of health service providers of different categories were conducted and additional information on records also reviewed. The results revealed that there was high level of awareness and knowledge of SP as an intervention for malaria prevention during pregnancy. However, negative factors like staff attitude towards clients, mix understanding of correct doses and interval, poor quality of services, and distance to health facilities, lack of proper education as well as culture hindered pregnant women from taking the drug leading to high dropout rate. The study posits that the trend is likely to continue unless a clear policy governing the IPT is efficiently implemented.
\end{abstract}

Keywords: Intermittent Preventive Treatment, Sulfadoxin- Pyrimethamine, Pregnancy, Malaria, Scaling up

\section{Introduction}

Every year, more than 30 million women become pregnant in malaria-endemic areas of Africa, causing up to 10,000 maternal deaths (WHO, 2010). The most common species that causes malaria in sub-Saharan Africa (SSA) is Plasmodium falciparum, and pregnant women are the most susceptible to this (ibid). It has been estimated that $75,000-200,000$ infant deaths are caused by malaria infection in pregnancy (Steketee et al. 2001). It is assumed that most pregnant women living in areas of high malaria transmission have malaria parasites in their blood or placenta, whether or not they have symptoms of malaria. Malaria infection during pregnancy increases the risk of maternal anemia about $2 \%$ to $15 \%$, Low birth weight (LBW) of $8 \%$ to $14 \%$, prematurity of $8 \%$ to $36 \%$ and intrauterine growth retardation (IUGR) of $13 \%$ to $70 \%$ (WHO, 2000).

\subsection{Fighting the Malaria Disease}

In 1998, the WHO, United Nations Children's Fund (UNICEF), the United Nations Development Programme (UNDP) and the World Bank (WB) established the Roll Back Malaria (RBM) programme with the sole aim of halving the malaria burden by 2010. Initially, in June 1997, Heads of states of Africa had met in Harare and issued a declaration on malaria prevention and control in the context of Africa's economic recovery and development. Following this, 44 Head of States and government delegations on the $25^{\text {th }}$ April 2000, adopted the Abuja Declaration on Roll Back Malaria (RBM), with a clear objective of ensuring that $60 \%$ of pregnant women and children under five, who are vulnerable and susceptible, have access to Insecticide Treated mosquito Nets (ITNs) and prompt effective treatment of malaria by the year 2005 (WHO, 2000). 
Since then, Ghana has made efforts to support the international fight to control malaria under the Roll Back Malaria (RBM) initiative. The RBM partnership has expanded with a range of organizations at global, regional and country levels. The partnership includes malaria endemic countries, United Nations (UN) organization, World Bank (WB) bilateral and multilateral development agencies especially the Global Fund, UNICEF, Non-governmental Organizations (NGOs) and Private Sector (Sachs, 2003).

A 2003 Ghana Demographic and Health Survey (GDHS) revealed that malaria is endemic in Ghana and continues to be a major concern of public health. Malaria among pregnant women accounts for $13.8 \%$ of all out-patient Department (OPD) attendance, $10.6 \%$ of admissions, $9.4 \%$ of deaths and presents adverse social and economic consequences for both mothers and babies. Previously, it was recommended that chloroquine should be the first treatment option by pregnant women as anti-malarial drug at ANC. The effectiveness of this strategy was short lived due to poor compliance and increasing resistance from plasmodium faliciparum, the causative agent (Sirima et al., 2003).

With the fact that résistance to chloroquine was becoming increasingly high the World Health Organization (WHO) in 2010 formulated new Guidelines to cover the diagnosis and treatment of uncomplicated and severe malaria caused by all types of malaria, including in special groups (such as young children, pregnant women and HIV/AIDS patients), in travellers (from non-malaria endemic regions) and in epidemics and complex emergency situations. The policy is to be implemented in all malaria endemic countries recommending that each pregnant woman should at least receive two doses of IPT after quickening (16weeks of pregnancy). This led to the WHO African Regional Office (AFRO), recommending the three-pronged approach using ITNs, IPT with SP and proper case management of malaria infection among all pregnant women in Africa especially countries with stable malaria.

\subsection{Intermittent Preventive Treatment during Pregnancy}

Intermittent preventive treatment is the administration of full treatment doses of an effective, preferably one-dose anti-malaria drug for the prevention of malaria at predefined intervals. WHO recommends that all pregnant women in areas of stable malaria transmission should receive at least two doses of IPT with SP after quickening (first movement of the fetus) during regular antenatal clinic (ANC) visits, which should not be given more frequently than one monthly ( WHO 2004).

The delivering of IPT with each routine of ANC visit will ensure that a high proportion of women receive at least two doses. Currently, the most used drug for IPT is SP because of its safety in pregnancy, effectiveness in reproductive-age women and feasibility for use in programs, since it can be delivered as a single dose treatment under observation by health worker. Fortunately in Ghana, over $90 \%$ of pregnant women attend antenatal clinic once before delivery which is an opportunity to administer the new anti-malaria drug at the health facility (GHS/NMCP, 2005). High antenatal attendance is not sufficient to ensure high IPT coverage. Some of the challenges with IPT distribution such as staff shortage, poor drug supply, poor access to ANC and poor staff attitude are some of the problems (Hill and Kazemba, 2006).

Current scientific evidence suggests that at least two doses of IPT with SP are required to achieve optimal benefit in most women. In settings with human immunodeficiency virus (HIV) prevalence in pregnant women greater than $10 \%$ it might be more cost-effective to treat all women with a three -dose regimen than to screen for HIV and provide this regime only to HIV-infected women (WHO 2000). HIV infection diminishes a pregnant woman's ability to control Plasmodium faliciparum infections (Steketee et al., 1999).

Based on empirical evidence from THE World Health Organization, most countries are distributing SP to pregnant women regardless of their HIV status to receive two doses of SP during pregnancy (WHO 2003). In confirmation of this, a comparative study conducted in Mali, proved that IPT with $\mathrm{SP}$ is more efficacious than weekly chloroquine chemoprophylaxis in preventing the adverse consequences of malaria in pregnancy (Kayentao et al., 2005).

In addition to IPT with SP for prevention of malaria during pregnancy, a number of studies have shown that Insecticide Treated Nets (ITNs) provide a varying degree of protection against malaria morbidity, anemia and low birth weight (Heggenhougen et al., 2003). In a trial of untreated bed nets in The Gambia, nets were found to reduce the number of infective bites from malaria (Snow et al., 1993). In subsequent studies, it was demonstrated further that use of ITNs in pregnancy reduces maternal parasitaemia, anemia premature deliveries, increases mean birth weight and subsequently reduces neonatal and infant mortality (Dolan et al., 1993; D’ Alessandro et al. 1995).

\subsection{Anti-Malaria Drug Policy for Ghana}

In 2003, the Ministry of Health (MOH) reviewed the guidelines for malaria prevention during pregnancy to bring it in line with the evidence associated with resistance and poor compliance of chloroquine. The most effective intervention, instead of the traditional weekly chloroquine given in the past, is the single dose of (SP) Sulphadoxine $500 \mathrm{mg}$ plus Pyrimethamine $25 \mathrm{mg}$ (Fansidar), three tablets in second trimester and repeated in the third trimester is adopted by many countries.

The Ghana Anti-malaria drug policy states that at least three (3) doses of SP shall be administered to pregnant women without HIV starting at quickening at least one month apart. The last dose shall be given at least one month before delivery (Anti-Malaria Drug policy for Ghana, 2004). The major aim of this intervention is to prevent the parasites from attacking the placenta so that the fetus can develop normally. As a result, the guidelines for malaria prevention in pregnant women were changed from chloroquine 
chemoprophylaxis to IPT using SP. The drug is to be administered under the supervision of a qualified health worker "Directly Observed Therapy (DOT)" (Anti-malaria Drug Policy for Ghana, 2004.)

\subsection{Implementation of the Anti-malaria drug policy in Upper West Region}

Malaria continues to be a major cause of morbidity and mortality in the UWR of Ghana (GHS, 2005). The implementation of the new drug policy in two districts (Jirapa/Lambussie and (Nadowli) on pilot bases since 2004, has met a lot of challenges in the region. Notwithstanding, the scaling up in the other six (6) districts continued. Interestingly, statistics from health facilities indicate that there is high drop-out rate of IPT with SP of between the first subsequent doses in all the districts (GHS, 2005). In 2005, 9697 pregnant women to the first round of IPT with SP but only 6826 took the second dose and the figure further reduced to 4027 in all districts in the region including the Wa municipality, see table 1 .

The question then is what has accounted for the high drop-out? Searching for the answer is the focus of the study.

Table 1. Administration of Sulphadoxine-Pyrimethamine (SP) to Pregnant Women by Districts, UWR, 2004 - 2005.

\begin{tabular}{lllllll}
\hline \multirow{2}{*}{ District } & $\mathbf{2 0 0 4}$ & & \multicolumn{5}{c}{$\mathbf{2 0 0 5}$} \\
& IPT1 & IPT2 & IPT3 & IPT1 & IPT2 & IPT3 \\
\hline Jirapa/Lamb & 2,577 & 1,066 & 782 & 4,003 & 3,217 & 2,377 \\
Nadowli & 1,645 & 1,094 & 632 & 2,800 & 2,586 & 1,218 \\
Sissala East & - & - & - & 343 & 94 & 0 \\
Sissala West & - & - & - & 393 & 158 & 54 \\
Wa East & - & - & - & 206 & 165 & 122 \\
Wa Municipal & - & - & - & 2,050 & 206 & 77 \\
Wa West & & & & 66 & 20 & 9 \\
Total & 4,222 & 2,110 & 1,414 & 9,697 & 6,826 & 4,027 \\
\hline
\end{tabular}

Source: GHS/UWR, Annual Report. 2005

\section{Methodology}

\subsection{Study Area}

There are six (6) government health facilities and one government referral hospital in the Wa Municipal, six private facilities -5 clinics and 1 private Maternity Home. There are numerous drug-shops, Traditional Trained Birth Attendance (TBAs) with few pharmacy shops who provide health care in the municipality.

Health services in the Municipality are delivered along the three-tier primary health care system; the community level made up of traditional healers, traditional birth attendants, chemical sellers and village health volunteers, level B (health centre) is responsible for providing public health and curative services which and above that antenatal services are provided, and where pregnant women can access IPT with SP. The referral point is level $\mathrm{C}$ where cases are sent for further management.

\subsection{Study Design}

The study was a cross-sectional descriptive one conducted to assess factors leading to the high drop-out rate in the use IPT with SP as first line malaria prevention among pregnant women. Both qualitative and quantitative methods were used to collect data including Focus Group Discussions, key informants Interviews and review of secondary data.

The team moved together in the entire sub-municipal unit until the work was completed. Preceding the field work, staffs of the selected facilities were briefed about the impending activities. The discussions centred on the aim and objectives of the study and also solicit for the consent of community and opinion leaders although individual consent was sought from participants and ethical issues clarified. Community entering was facilitated by health workers who worked directly with the communities and mobilized pregnant women and postnatal mothers for the discussions.

The community health nurses who work directly with the communities helped mobilized the pregnant women and postnatal mothers for the discussions. Participation was voluntary as respondents were asked for their consent to take part in the FGDs. Moderation of the FGDs was by the principal investigator with the support of research assistant experienced in qualitative data collection and also fluent in the local dialect. Notes were taken and discussions recorded on tapes. Transcriptions of the FGDs and the notes were re written out boldly and neatly and stored in clear bags and labelled for verifying the content as well as the validity of opinions. The key informant interviews and records reviews were conducted by the principal investigator and documented.

\subsection{Study Population}

Study participants were pregnant women, postnatal mothers and health workers of various categories; municipal public health nurse (MPHN), the pharmacist in charge of regional medical stores and the regional malaria coordinator who are directly involved in the implementation of the drug distribution were the respondents of this study.

\subsection{Sampling}

In addition to the regional hospital four sub-municipal and two communities were randomly selected out of the 6 sub-municipal health facilities for the focus group discussions. A list of 17 categories of health service providers were selected for the Key Informant interviews with in the study area.

\subsection{Data Management and Analysis}

Data from the semi-structured and face-to-face interviews was analysed using SPSS. The data from the focus group discussions was summarized according to the two groups, that of pregnant women and the postnatal mothers. Key informants responses and contributions were identified and synchronised with FGDs. The main variables in the question 
guide included knowledge of malaria, awareness of IPT with SP, factors preventing pregnant women from accessing SP, challenges faced in the distribution of SP and suggestion for the improvement of were coded for easy reference and compilation. Notes taken and cassettes used during the discussion were reviewed and transcribed from the local dialect (Waali) into English and systematically analysed for commonalities. Data from health facilities records were reviewed to find out about drugs supply. Lastly, the data was contrasted and compared with the themes across the interviews and FGDs since both tools were exploring the same thematic areas.

\subsection{Ethical Considerations}

Consent was sought and permission approved from Research Unit of the Regional Health Administration and Municipal Directorate by applying well ahead of time. Regular interpersonal communication was established to get approval and support. Community entering skills was implored by dialoguing with community members and health workers for the appropriate time for interviews and Focus Group Discussions (FGDs) was ensured. The consent of all persons that were selected was sought as to their willingness to participate. They were made aware that they could opt out if they did not wish to participate and this will not affect their ability to benefit from any health service. All those who chose to participate were assured that any information provided will be kept confidential. And that information given was to help authorities improve upon health services.

\section{Results}

The findings of this study were obtained from collation of data from six focus group discussions made up of 4 pregnant women's group and two groups of postnatal mothers. Seventeen key informant interviews were conducted at the health facilities and respondents included midwives, community health nurses, public health nurse, malaria coordinators and a regional pharmacist in charge of regional medical stores (RMS). Records of the municipal Health Directorate, Wa regional hospital, Busa and Charia health centres as well as Pregnant women's ANC cards were reviewed. The findings are presented under the following themes: background informants of respondents, knowledge of IPT with SP, services rendered in relation to SP distribution, factors preventing pregnant women from taking the full doses of the drug (SP), challenges and suggestions for improvement of SP uptake and distribution.

\subsection{Background Characteristics of Respondents}

The age group of the respondents ranged between 19-60yrs. Sixty-nine (69) participants took part in the research. Out of the 69 respondents who participated, $52(75 \%)$ were involved in the focus group discussions females and $17(25 \%)$ were key informants. Pregnant women were $(58 \%)$, postnatal mothers were $(23 \%)$ and $19 \%$ were other hospital staff. The seventeen (17) key informants interviewed included medical officers in-charge of the sub districts, midwives, community health nurses, regional malarial control officer and the Pharmacist in charge of the regional medical stores in the municipality.

Out the 69 interviewed (67\%) were illiterates while (30\%) had some level of education ranging from Junior Secondary School (JSS) to Senior Secondary School (SSS) and (3\%) finished tertiary level of education. The high level of illiteracy $(67 \%)$ clearly shows that health education should be done in the local dialect.

\subsection{Knowledge of Malaria and IPT with SP among Pregnant Women}

In all the focus group discussions, a significant number of women had knowledge on the causes, signs and symptoms of malaria. They mentioned dizziness, bodily weakness, headache and nausea as some of the signs and symptoms. The common local terminology used to describe malaria throughout the study was "Maahu" in the local dialect (Waali). In addition another woman said "those small, small insects found in swampy and dirty places cause malaria" (FGD no 1, Respondent number 5 "R5"). This expression demonstrates the level of awareness among respondents about malaria. The symptom that was frequently mentioned was fever, accompanied by increase in body temperature. Other respondents also mentioned drinking plenty of water, vomiting and excessive sleeping as some of the symptoms. There was no difference between the postnatal mothers and the pregnant women in mentioning the signs and symptoms.

Among the key informants there was adequate knowledge about SP as common prophylactic distributed to pregnant women at all health facilities in the Wa Municipality. "It has come to replace chloroquine and gradually $S P$ and artesunate-amodiaquine will be the drug of choice to manage malaria" (Key Informant-KI no.12). On exploring the anti malaria drug distributed at the various health facilities, artesunate-amodiaquine and quinine were the common drugs used in the treatment of malaria and Malafan ${ }^{\circledR}$ or Fansidar ${ }^{\circledR}$ (SP) distributed in prevention of malaria in pregnancy. It was further explained that almost all health workers have had training on the new drug policy.

\subsection{Prevention of Malaria}

It was well-know among most of the community respondents that keeping the environment clean, sleeping under ITN and SP were some of the preventive measures to control malaria during pregnancy. At the Wa regional hospital, few of the FGDs respondents knew that SP was a prophylactic for prevention of malaria and could mention the name as well as describe it "they are three tablets and look like paracetamol and we take it with water while the nurses observe us." (FGD1. PWR8). Exploring for other ways of preventing malaria most of it was mentioned clearing of bushes around homes, draining of stagnant water, 
arrangement of clothing in our rooms and organising clean up campaign in our communities. Despite the knowledge malaria prevention using SP among pregnant women is very poor.

\subsection{Perception of SP First Dose and Subsequent Ones}

In all the FGDs conducted to assess opinion about all doses, three quarters of the participants were not sure about the number of times to take or when exactly to return for the next dose but, said three tablets should be taken each time, few mentioned that SP should be taken two, three or more times. "I remember my friend during her pregnancy took the drug more than four times but for delivery she would have taken more of the drug because it is good" (PNM FGD $R 5)$.On reasons, it was stressed that the health workers know what is good for pregnant women and their babies, knowledgeable about health problems and can explain issues to us. This shows that community members are ready to adopt information and change behaviour if the enabling environment is put in place.

Ten out of the seventeen key informants mentioned that some health workers do not explain at least the benefits of drug very well to the understanding of the pregnant women especially primgravidas. "Some of us are always rushing with the clients, there is no time to explain important matters concerning their health"(KI 1).

\subsection{Factors Preventing Pregnant Women from taking SP}

On factors that prevent pregnant women from taking the subsequent doses; most of respondents from the focus group discussion mentioned that distance to the health facilities is difficult for pregnant women to walk. The cheapest means is the government transport called "Metro Mass" transport, which is not regular.

This was affirmed by the key informants as one of the obstacles that prevent pregnant women from taking their subsequent doses. "The Metro Mass buses must move to the villages to help the poor pregnant women, at times one has to come and sleep with a relative in order to attend antenatal clinic (FGD 2.R8). In addition to distance to health facilities, majority of KIIs mentioned poverty as a one of the factors. "I strongly believe the inaccessible roads coupled with the rains also prevent health workers from reaching pregnant women to distribute SP" (KI No.7). One of the barriers associated with the low uptake is health workers attitude, information is not usually disseminated very well to clients. Most of the participants in all the FGDs felt staff attitude prevents most of the pregnant women from taking the drug. In addition, they are shouted at when there is the need for a clarification of issues concerning SP uptake. A few of the key informants agreed with this. "At times the nurses don't tell you the effects of the drug in individuals. When you complain they don't explain things for you to understand but rather shout on you to go and take it. So others will simply go away with the drug and throw it away" (FGD 3 R8). Few respondents of the focus group said they had no reaction rather they feel well and can eat also.

Majority of the postnatal FGDs were of the opinion that money is a hindrance, "From my house to the Clinic is five hundred cedis and another five hundred cedis back. If I don't have the money, I cannot come". In a follow up comment, a pregnant woman who had receipts to back her point mentioned that "At the Wa Regional Hospital for instance Antenatal card is fifteen thousand cedis, laboratory investigation is $\$ 85,000$ (with receipt to back her point) and so if you don't have Health Insurance and no cash, what can you do?

Few believe the mutual health insurance (MHI) process is slow and not allowing people to benefit from it. A nineteen year old pregnant woman stated that "There was an instance where a colleague of mine- pregnant girl with Health Insurance was refused laboratory test". Most of the respondents in the study believe the District wide Mutual Health Insurance (MHI) process is slow and not allowing people to benefit from the services.

Most FGDs participants and KIIs respondents were of the view that late reporting was a problem as pregnancy is concealed till it is at the advance stage leading to most of them taking just one dose. During discussion migration down the southern part of the country was also a factor. " $M y$ in law, since we have one rainy season in this part of the Country, most couples migrate down South to farm". (KI No. 2). Interestingly, another key informant was of the view that "some pregnant women are facility "flirts" ....they move from facility to facility seeking health service" (KI No. 4).

In all, FGDs and key informants perceived religion (Moslem) as an obstacle in the municipality even if one is pregnant. This issue was further probed to find out why pregnant women should fast, "if you fast you will get blessings for yourself and the unborn child, another thing is that if you don't do it with others during the fasting period it will still be waiting for you, so why don't I try and do it and get it off my head"

Few of the key informants mentioned poor documentation, staff attitude, ignorance and peer influence and side effects even though not reported for fear of not attended to as some of the reasons that prevent pregnant women from accessing the services of SP.

Secondary data reviewed at the Wa municipality confirmed that, there has been increase uptake of IPT with SP. Table 2 compares records of 2005-2006 distribution of SP among pregnant women.

Table 2. IPT with SP Distribution to Pregnant Women in Wa Municipality.

\begin{tabular}{lllllll}
\hline & $\mathbf{2 0 0 5}$ & & & 2006 & & \\
& IPT1 & IPT2 & IPT3 & IPT1 & IPT2 & IPT3 \\
\hline Total & 268 & 157 & 73 & 3040 & 2325 & 1692 \\
\hline
\end{tabular}

According to key informants, majority of the respondents were aware of the new drug distribution but still do not believe it can prevent malaria. 
"I remember my friend during her pregnancy took the drug more than four times... but for delivery she would have taken more of the drug because it is good"'(PNM FGD R5)

\subsection{Quality of Services Rendered in Relations to SP Distribution}

About services rendered concerning SP, most of the participants mentioned that apart from ensuring that they take the drug while the nurses observed them, no advice or counselling about the drug was given them. "At times the nurses will give you the drug and ask you to go and take without explaining anything to you...if something happens to you whose fault"

A few said physical examinations, laboratory investigations and treatment of minor illness make them happy. On the contrary the majority of the key informants mentioned other services rendered in relation to SP as counselling of pregnant women on side effects of SP; health staff also advised them to come for follow up for the other doses and management of side effects. Few of the Key informants were of the opinion that some staff attitude is really preventing the pregnant women from accessing the services. "For example if a pregnant woman comes from far off community and the nurse is about closing she wouldn't have the patience to attend to the poor woman who has may be walked for more than five kilometres or simply say the drug is finish". (KI 14). This shows that poor communication skills among health service providers especially midwives in handling pregnant women who attend ANC.

Whether respondents will recommend the drug (SP) to pregnant women, all unanimously affirmed that they will (laughing) reason being that "it is health workers who know what is good for us, nurses should be patient to explain the side effects of the drug and not enforce all pregnant women to take the drug without listening to the person's complain". (FGD 1 R3)

\subsection{Availability of Sulfadoxine-Pyrimethamine}

This study shows in all six FGDs and key informants interview that, irregular supply of the drug since the implementation in 2005 is not motivating some of the women to access the services.

Most of the facilities where data concerning SP distribution was available revealed irregular supply of SP towards the latter part of 2005. Some sampled antenatal cards of multgravida confirmed that during their previous pregnancy SP doses column was either blanket or was given just the first dose.

"If I hear there is something good for pregnant women and I come and don 't get it, will I come again".(FGD 1 R 5). In ensuring quality of services at facilities there is the need for management to regularly supply logistics and motivate health workers to be at post.

\subsection{Challenges}

Asked about challenges faced with the distribution of SP, all key informants stated that irregular and inadequate supply of SP is the major challenge. "Sister supply is inadequate, irregular and at times not available at the national level........... Quantities supplied to the region should increase because of distance from the main source of supply, another problem is the procurement law it is so complex" (Key Informant No.17). This area was further explored to find out how complicated the act work, it was said that the process was the problem.

In confirmation to FGDs distance was stressed as a challenge to seeking access to health facilities in relation to distribution of SP within the municipality not forgetting the staffing situation.

\subsection{Adequate Staffing}

Another challenge that most of the Key informants mentioned were too many forms to fill coupled with the shortage of staff making documentation very poor. "Presently we are two technical staff and one unskilled worker providing services in this health facility. I am alone now, there is a woman in labour, there are patients to be treated so, at the end of the day you are tired and therefore poor documentation." (KI 13)

Another area is unskilled workers who can not perform certain duties which are very vital in the delivery of services. This contributes to poor quality of services and not attracting clients and patients not accessing the health facility.

\subsection{Suggestions for Improvement}

A significant number of KIs are of the opinion that midwives, chemical sellers and community-based agents (CBAs) should be trained in the distribution of SP, further more it was suggested that the requested amount of drug should be supplied to ensure regular availability of the drug at the health facilities. In addition key informants were of the opinion that SP should be included in the regular drug supply list. Another area that needs attention is intensification of health education using the local dialect through the media (Radio stations).

Similarly, most of the focus group participants agreed that once the CBAs are the first point of call in health matters SP should be added to their package. In addition, all Traditional Birth Attendance, (TBAs) Village health volunteers and community health agents should be given some basic training in the distribution of the drug.

"Let me add that the volunteers should be given some incentives to motivate them since they are not paid. (FGD3 PNM no.5) Another postnatal mother, "I agreed with what my friend has said, during raining season whiles others are on their farms the volunteer will be busy mobilising community members for health activities".

Few suggested that the volunteers should be committed to the work and that once the community has chosen them to help health workers in health issues they should accept it. Records review at all facilities where the study was conducted showed that either there were newly created 
health facility and no full documents for SP for the year 2005 , or poor documentation of information.

Table 3 SP Supply to Wa Sub Municipality. 2005-2006

\begin{tabular}{lll}
\hline & $\mathbf{2 0 0 5}$ & $\mathbf{2 0 0 6}$ \\
\hline Facility & Quantity supplied & Quantity supplied \\
Bamahu & $*$ & 700 \\
Busa & $-\overline{450}$ & 950 \\
Charia & 501 & 800 \\
Kambali/Nakori & - & 900 \\
Wa Central & 1,047 & 1,650 \\
Wa Regional Hospital & 1,752 & 5,950 \\
Total & 3,750 & 12,050 \\
\hline
\end{tabular}

* Facility was not operational

The above table shows the improvement of SP supply in 2006, this could be due to the support UNICEF gave during the period.

\section{Discussion}

Decision makers and programme officers have made efforts to identify ways of improving access and compliance to malaria prevention intervention as a priority in the public health sector. This is because malaria is the leading cause of morbidity and mortality among pregnant women and children under five years.

This study has given insight into barriers preventing pregnant women from using SP leading to high drop out rate Although findings cannot be said to be representative of the whole municipality but has given some indication for the poor performance of SP distribution in the Wa municipality. It is important to note that number of times pregnant woman need to take SP acts as a baseline for prevention of malaria; first it affects the acceptance and it is important to design health education messages involving community members so as to create the enabling environment that would stress on the benefits of taking the required doses and at what interval. The awareness of using SP for the prevention of malaria in pregnancy is high among women in the Wa municipality. Some of them did not know the name of the drug which is used at the antenatal clinic, may be because of the various names Fansidar, ${ }^{\circledR}$ or Malafan ${ }^{\circledR}$ (SP) but majority could describe it very well. This finding is encouraging and may have an impact on the uptake of SP to reduce drop-out rate.

However, pregnant women have mix information on the number of times and actual doses to take SP. If important messages are not designed properly and disseminated as expected it will surely have an effect on good programmes. This could also influence personal experience, traditional beliefs, economic, social factors and interaction within the community and health service providers out put. Intensifying health education at the health facilities using the local terms to explain prevention of malaria in pregnancy will help. According to Ahorlu, 1997, understanding people's knowledge about malaria and the factors that influence these perceptions is central to increasing successful malaria prevention intervention. In addition, a team in Mukono district, Uganda on perception on use of SP in pregnancy revealed that negative attitude of health workers was the main barrier especially midwives and another been distance (Anthony M.K et al.,2005).

The role of service providers in the distribution of SP plays a vital role as revealed by the study quality of services rendered forms the bases of interactions between communities and health facilities. Majority of the respondents in the discussions were of the view that distributing the drug is important but having the patients' to explain issues concerning benefits, consequences and steps to take incase of reactions are not usually discussed. The problem of poor nurse-patient relationship is clearly a complex one and multiple solution is required to handle this problem. This perception forms the basis of seeking care at health facilities and is a challenge to the implementation of the malaria prevention programme. A study has proven quality of health services from a community perspective in Soroti district of eastern Uganda that inadequate services provided by health workers especially midwives was due to non adherence of guidelines, confirming lack of quality services at our health facilities (Kaye. 2000).

Quality of service plays a vital role in delivery of health service so therefore service providers need to be caring and build trust between clients and themselves to encourage them access the services. The present study has shown late attendance as a major cause of pregnant women not receiving the full dose of SP. Cultural beliefs, that pregnancy in the early stages should not be known until advanced and this tradition is deep rooted in Wa municipality. A significant number of the key informants affirmed this further saying that there is the belief that, some people have evil eyes that can see through the human body especially the pregnant woman therefore they hide the pregnancy until it is advance before attend clinic. Findings in two Kenyan studies which documented low compliance with IPT and reason for non-compliance was late antenatal attendance (Guyatt et al., 2004; van Eijk et al., 2004). It is clear from the study that education with well designed messages for community members will be beneficial.

This study has revealed that inadequate supply of logistic is a major hindrance to health seeking. It would be appropriate that during performance meeting, service providers and stakeholders have frank discussion that will create an enabling environment for clients and patients to access health at our facilities. Nevertheless, the study discovered that partnership with United Nations Children Emergency Fund (UNCEF) enable the metropolis accessed some quantity of SP (data not available) that has seen significant increase of SP supply and subsequent uptake by pregnant women. This clearly demonstrates that there are other partners who have interest in supporting the implementation and expansion of the programme that will reduce the burden of malaria. Some of these public-private partners just to mention a few are; Global Fund for AIDS, Tuberculosis and Malaria (GFATM), United States (US) Presidential Malaria Initiative (PMI), the Malaria Vaccine 
Initiative (MVI) supported by the Bill and Melinda Gates Foundation are areas that needs collaboration. This will ensure regular supply of SP and remove all obstacles that will serve as a disincentive in the performance of health staff and build trust between community members and health service providers. Perry 1999 argued that delivery of proper health care is not only the supply of care but also the acceptance and use of services by the patients and clients.

Further more in this study; it was eminent that pregnant women who accessed the first dose of IPT during routine ANC complained of long distance to health facilities and another factor that prevents them from taking the subsequent doses subsequent. Sending health services to the door steps of community is still a hindrance to the section. Thought policies have been documented to this effect. For example heat for all by the year 2000 , is still a mirage. Similar findings where quality of ANC affected access and use of services has been documented in Uganda and elsewhere (Ndyomudgyenyi et al.,1989; Hortz et al., 2004; Guyatt et al; 2004; Ammoti-Kaguna \&Nuwaha, 2000; Kaye, 2000).

An important factor discovered by this study which prevents the up take of SP after the first dose is money. Despite the fact that District Mutual Health Insurance Scheme (DMHIS) is being implemented to increase access to services, there are challenges with the process either health workers do not recognize it or the process of getting identity card is slow. (Gh.HI.,LI 2005). Finance should not be a hindrance for pregnant women to access SP services within the health facilities, but the high bus fares as well as paying for registration cards and other services underscore that poor finances are significant contributors to poor uptake and high drop-out. The study has revealed that community members are beginning to realize the benefit of health insurance and will only seek health services if they are sure of financial relief from the mutual health insurance. This is an area that needs to be explored for proper intervention put in place.

The study had limitations; concerning the three doses which is well documented yet rationale for that decision of three doses by Ghana instead of the recommended two doses by WHO was a hindrance to this study. Efforts were made to contact people concerned about this policy but it proved futile. The Ghana Health Management Information systems play a vital roll in the health systems therefore the need to have a well structured system that will ensure proper documentation of information. Another limitation is that no study has been conducted except an evaluation of SP distributions one of the pilot districts making reviewing information difficult. Pregnant women were initially the target group for sampling but during data collection there was the need to randomly select postnatal mothers to fill the gap due to nurses strike action. Strike actions are becoming normal situations in Ghana which the government is aware so therefore measures been taken to solve these problems.

Continuous in-service training as a strategy will be useful in changing attitudes of staff that are currently practicing. In considering the training of community-based agents, traditional birth attendance and village health volunteers has its own implications. Either drugs will be over dosed to pregnant women or the period at which to administer the drug will be miss calculated and can lead to adverse consequence. Though community members trust their own members it is not feasible for these supporters of health delivery to be trained in this area because of the technicalities involved for example assessing the pregnancy for quickening.

\subsection{Conclusion}

Though this study had limitation, it has identified issues that need to be addressed. The drop-out rate within the Upper West Region starting with the pilot districts and scaling out to the other districts is well established. This study has shown that there is adequate knowledge of Sulfadoxine-Pyrimethamine for pregnant women in the $\mathrm{Wa}$ municipality. However, SP is perceived as routine drug not as preventive measure for protecting mother and fetus against malaria. In addition, there is limited knowledge among pregnant women on dosage and the number of times that SP should be taken.

Other factors identified that prevented pregnant women from accessing SP leading to high drop-out rate included, quality of services provided at the health facilities, culture as revealed in the study, reporting late for antenatal care, irregular supply of drugs, distance to health facilities coupled with bus fares, attitude of health staff-example shouting and scolding of clients and patients, mixed understanding of SP uptake, the everyday practices at health care facilities, perceived quality of care and poor public education among others. Health workers were also of the view that additional workload in terms of inadequate staffing; too many documentations to contend with and irregular supply of drugs and staff attitude as factors that contribute to pregnant women not accessing all three doses after the first dose.

\subsection{Recommendations}

Continuous in-service training using national guidelines for newly employed health staff and refresher training to upgrade the already trained health staff on IPT with SP programme will improve upon the delivery of services specially IPT. In addition, intensified health education by educators including health workers, using the correct updated information delivered in a culturally sensitive context; using appropriate media (Radio discussion using local language) and target groups could help bring the desired changes in the uptake of SP.

Integrating the new drug strategy with other antenatal activities and outreach services would be cost effective and also reduce the drop-out rate. For example Voluntary counseling and testing services rendered at the antenatal clinics.

The Community-based health planning and services (CHPS) programme should be encouraged to fill the gap 
between the community and health service delivery. Not forgetting regular home visits to identify pregnant women who default and educate those who do not know when and why the need to take the required dosage of SP and about the benefits to both mothers and their unborn babies.

At the national level, regular review of the current policy on drug procurement should be revisited; the process should involve relevant stakeholders to design an acceptable local policy that will facilitate the purchasing of SP at the local level. This will go far to prove that decentralization is practicable but not merely the making of decisions.

There is the urgent need for an in-depth research in the Upper West Region to ascertain the impact SP is making on pregnant and their babies.

Regardless of operational problems associated with the distribution of IPT with SP, the information about the new drug has been disseminated very well to the target group in the municipality especially of awareness of SP for the prevention.

\section{References}

[1] A.Mbonye, S.Neema, P. Magnusson, (.2005) Perceptions on use of sulfadoxin-Pyremithemine in pregnancy and the policy implications for malaria control in Uganda. Health Policy, Vol.77 279-289.

[2] Ministry of Health (2004) Anti-malaria Drug policy for Ghana.

[3] D'Alessandro et al. (1995) A comparison of the efficacy of Insecticide-treated nets and untreated nets in preventing malaria in Gambia children. Transactions Royal society of Tropical Medicine and Hygiene. 89: 596-8

[4] Dolan G, ter Kuile FO, Jacoutot V et al. (1993). Bed nets for the prevention of malaria and anemia in pregnancy. Transactions Royal society of Tropical Medicine and Hygiene 87: 620-6

[5] Ghana Statistical Service. (2003) Demographic Health Survey. Ghana.

[6] Ghana Health Service, (2005) Upper West Regional Health Report;.Ghana

[7] Ghana Health Service/National Malaria Control Programme;(2005) Intermitted Preventive Treatment (IPT) of Malaria in pregnancy; Training Manual for Health Providers:

[8] Heggenhougen, H.K., Hackenthal, V.\& Vivek, P.(2003) The Behaviour and Social Aspects of Malaria Control: An introduction and Annotate Biography. Special Programme for Research \& Training in Tropical Disease.

[9] Jenny Hill \& Peter Kazember; (2006) Reaching the Abuja target for Intermittent Preventive Treatment of Malaria in Pregnancyin African women: a review of progress and operational challenges. Liverpool School of Tropical Medicine, 11:409-418.

[10] Jirapa/Lambussie (2007) Evaluation of Intermittent Preventive Treatment. District health management team
(Yet to be disseminated) Ghana

[11] Kassoum et al (2005). Comparison of Intermittent Treatment with Chemoprophylaxis for the prevention of Malaria during pregnancy in Mali. The journal of Infectious Diseases, Vol.191, P 109-116.

[12] Kaye, D., (2000). Quality of midwifery care in Soroti district, Uganda. East Africa Medical journal 77,558-61.

[13] Ndyomugyenyi, R.,Neema, S., Magnussen, P.,( 1998). The use formal and informal services for Antenatal care and malaria treatment in rural Uganda. Health pol. Plan. 13, 94-104.

[14] RBM.WHO.INT http://mosquito.who.int Viewed on 22/ 03/ 07

[15] Rogerson, et al (1997) Intermittent Sulfadoxine-pyremithemine in pregnancy: Effectiveness against malaria morbidity in Blantyre, Malawi in $1997-99$. Trans.Roy.Soc. Trop.Med.Hgy. 549-553.

[16] Sachs J, Foreword (2003).Reducing malaria's burden. Evidence of effectiveness for decision makers. Global Health Council Technical Report.

[17] Shulman CE. Malaria in pregnancy: (1999) its relevance to safemotherhood programme on the outcome of pregnancy. Annals of tropical medicine and parasitology

[18] Sirima, S.B et al. (2003) Failure of chloroquine chemoprophylaxis programme to adequately prevent malaria during pregnancy in Koupela district, Burkina Faso. Clinical infectious diseases, 36 (11):1374-1382.

[19] Snow, RW, Rowan K.M, Lindsay S W, Green B M.(1988). A trail of bednets(mosquito) as a malaria control strategy in a rural area of the Gambia, West Africa. Transactions Royal Society of Tropical Medicine and hygiene, 82: 212-5

[20] Steketee et al. (1999) Impairment of a pregnant woman's acquired ability to limit Plasmodium falciparum by infection with human immunodeficiency virus type-1.American journal of tropical medicine and hygiene,,55(1 suppl.): 42-49.

[21] Steketee RW et al. (2001) The burden of malaria in Pregnancy malaria-endemic areas. American journal of tropical medicine and hygiene, 64) 28:35

[22] Van Eijk, J. G Ayisi, F. O. (2004). Effectiveness of Intermittent Preventive Treatment with Sulphadoxine -Pyrimethemamine for control of malaria in pregnancy in western Kenya: a hospital -based study. Trop. Med. Int.Health. Page 351.

[23] Wa Municipal (2006) Health directorate; annual report; Ghana

[24] WHO.(2004)The African summit on Roll Back Malaria, Abuja, Nigeria.200.,17(WHO/CDC/RBM

[25] WHO. (2010) A strategic framework for malaria prevention and control during pregnancy in the African region. Brazzaville, World Health Organizatio; AFR/MAL/04/01.

[26] WHO.(2000) Communicable diseases cluster. Severe falciparum malaria. Trans R Soc Trop Med Hgyg . 